\title{
Exclusion Statistics of Composite Fermions
}

\author{
Piotr Sitko \\ Institute of Physics, Wrocław University of Technology, \\ Wybrzeże Wyspiańskiego 27, 50-370 Wrocław, Poland.
}

\begin{abstract}
The exclusion statistics parameter of composite fermions is determined as an odd number $(\alpha=3,5, \ldots)$. The statistics of composite fermion excitations at $\nu=\frac{n}{2 p n+1}$ is rederived as $\alpha_{q e}^{C F}=1+\frac{2 p}{2 p n+1}, \alpha_{q h}^{C F}=1-\frac{2 p}{2 p n+1}$. The duality $\frac{1}{\alpha_{q e}(n, 2 p)}=\alpha_{q h}(n+1,2 p)$ is found. The distribution function for $\alpha=3$ is obtained.
\end{abstract}

\section{Introduction}

The exclusion statistics was proposed by Haldane [1] as generalization of Pauli exclusion principle. The idea of Haldane is to define the change in the available particle space when particles are added to the system (or removed):

$$
\Delta d_{i}=-\sum_{j} \alpha_{i j} \Delta N_{j}
$$

The generalization of the Haldane exclusion statistics was proposed by $\mathrm{Wu}$ [2] and others [3, 4, 5]. The idea of generalization is to divide the available particle space for smaller cells (let us say with $k$ states). The number of many-particle states is given by:

$$
\prod_{i}\left(\begin{array}{c}
d_{N_{i}}+N_{i}-1 \\
N_{i}
\end{array}\right)
$$


where $d_{N_{i}}=k-\alpha\left(N_{i}-1\right), \alpha=\alpha_{i i}$.

Johnson and Canright used the spherical geometry to find the statistics of Laughlin quasiparticles [6]. Analyzing numerical results they found the number of many-particle states of quasiholes (of the Laughlin $1 / m$ state, $m$ - odd number):

$$
\left(\begin{array}{c}
N_{e}+N_{q h} \\
N_{q h}
\end{array}\right)
$$

where $N_{q h}$ is the number of quasiholes, $2 S+1=m\left(N_{e}-1\right)+1-N_{q h}, 2 S$ is the number

of flux quanta piercing the sphere. According to the definition (2) $\alpha_{q h}=\frac{1}{m}$ [6]. The corresponding statistics parameter of Laughlin quasielectrons is $\alpha_{q e}=2-\frac{1}{m}$. Here, we perform similar analysis in order to determine the exclusion statistics of composite fermions.

\section{Composite fermions}

The exact diagonalization results for the sphere can be interpreted in terms of composite fermions [7] if the effective field $2 S^{*}=2 S-2 p\left(N_{e}-1\right)$ is introduced. The composite fermion approach predicts the same angular momentum shell for quasiparticles [7], however, the main role play composite fermions (the number of composite fermions equals the number of electrons $N_{C F}=N_{e}$ ). The Eq. (3) is:

$$
\left(\begin{array}{c}
2 S^{*}+1 \\
N_{q h}
\end{array}\right)
$$

and equals

$$
\begin{gathered}
\left(\begin{array}{c}
N_{C F}+2 S-m\left(N_{C F}-1\right) \\
N_{C F}
\end{array}\right) \\
=\left(\begin{array}{c}
2 S+1+(1-m)\left(N_{C F}-1\right) \\
N_{C F}
\end{array}\right) .
\end{gathered}
$$

According to the definition (2) the statistics parameter of composite fermions $\alpha_{C F}=m$ (odd number $\alpha_{C F}=3,5, \ldots$, for $\alpha=1$ one has fermions).

$\mathrm{Wu}$ et al. [2] found the distribution function of $\alpha$-particles. The duality between $\alpha$-particles of $\alpha=1 / m$ (holes) and $\alpha=m$ was noticed by Nayak and Wilczek [3]. Our analysis reflects the duality between Laughlin quasiholes and composite fermions.

The distribution function is given by the set of equations [2]:

$$
\begin{gathered}
n_{i}=\frac{1}{w+\alpha} \\
w^{\alpha}(1+w)^{1-\alpha}=\xi
\end{gathered}
$$




$$
\xi=e^{\frac{\epsilon_{i}-\mu}{k T}}
$$

For example, for $\alpha=3$ the solution is

$$
w=\frac{1}{s_{+}+s_{-}-\frac{2}{3}}
$$

where

$$
s_{ \pm}=\left[\frac{1}{2 \xi}+\frac{1}{27} \pm \sqrt{\frac{1}{\xi}\left(\frac{1}{\xi}+\frac{1}{27}\right)}\right]^{\frac{1}{3}} .
$$

It is interesting to consider also the composite fermion excitations. Let us consider the filling $\nu=\frac{n}{2 p n+1}$, then one gets $n$ filled shells (in the field $2 S^{*}=2 S-2 p\left(N_{e}-1\right.$ ) the degeneration of the $n-t h$ effective shell is $\left.2 S^{*}+2 n-1\right)$. When one creates $N_{q e}$ quasielectrons (of the state $\nu=\frac{n}{2 p n+1}$ ) in the $(n+1)$-th level, the number of many-particle states is

$$
\left(\begin{array}{c}
2 S_{q e}^{*}+2 n+1 \\
N_{q e}
\end{array}\right)
$$

and

$$
2 S_{q e}^{*}=-\frac{2 p}{1+2 p n} N_{q e}+\frac{2 S-2 p n^{2}+2 p}{1+2 p n} .
$$

If quasiholes are present in the $n$-th level then

$$
\left(\begin{array}{c}
2 S_{q h}^{*}+2 n-1 \\
N_{q h}
\end{array}\right)
$$

and

$$
2 S_{q h}^{*}=\frac{2 p}{1+2 p n} N_{q h}+\frac{2 S-2 p n^{2}+2 p}{1+2 p n} .
$$

Hence, according to the definition (2)

$$
\begin{gathered}
\alpha_{q e}=1+\frac{2 p}{1+2 p n}, \\
\alpha_{q h}=1-\frac{2 p}{1+2 p n}
\end{gathered}
$$

as was first obtained in Ref. [8]. For $n=1$ one gets the Laughlin states $\frac{1}{m}=\frac{1}{2 p+1}$. One can notice the duality between quasielectrons of the state $\frac{n}{2 p n+1}$ and quasiholes of the state $\frac{n+1}{2 p(n+1)+1}$ :

$$
\frac{1}{\alpha_{q e}(n, 2 p)}=\alpha_{q h}(n+1,2 p)
$$

For example, consider quasielectrons at $1 / 3$ and quasiholes at $2 / 5$. 


\section{Conclusions}

It is found that composite fermions can be described within the generalized exclusion statistics as particles with statistics parameter $\alpha$ being an odd number. Also, the exclusion statistics of composite fermion excitations is rederived as $\alpha_{q e}=1+\frac{2 p}{1+2 p n}, \alpha_{q h}=1-\frac{2 p}{1+2 p n}$. The duality $\frac{1}{\alpha_{q e}(n, 2 p)}=\alpha_{q h}(n+1,2 p)$ is found.

This work was supported by KBN grant No. 2 P03B 11118.

\section{References}

\section{References}

[1] F. D. M. Haldane, Phys. Rev. Lett. 67 (1991) 937

[2] Y-S. Wu, Phys. Rev. Lett. 73 (1994) 922

[3] C. Nayak, F. Wilczek, Phys. Rev. Lett. 73 (1994) 2740

[4] A. K. Rajagopal, Phys. Rev. Lett. 74 (1995) 1048

[5] A. Dasniéres de Veigy, S. Ouvry, Phys. Rev. Lett. 72 (1994) 600

[6] M. D. Johnson, G. S. Canright, Phys. Rev. B 49 (1994) 2947

[7] X. M. Chen, J. J. Quinn, Solid State Commun. 92 (1994) 865

[8] S. B. Isakov, G. S. Canright, M. D. Johnson, Phys. Rev. B 55 (1997) 6727 